\title{
Overexpression of miR-422a inhibits cell proliferation and invasion, and enhances chemosensitivity in osteosarcoma cells
}

\author{
MINGJIANG LIU $^{1}$, HE XIUSHENG $^{2}$, XIANGJUN XIAO $^{1}$ and YICHUN WANG $^{3}$ \\ ${ }^{1}$ Department of Hand Surgery, Affiliated Nanhua Hospital, University of South China, Hengyang, Hunan 421002; \\ ${ }^{2}$ Cancer Research Institute, University of South China, Hengyang, Hunan 421002; \\ ${ }^{3}$ Department of ICU, Hunan Cancer Hospital, Changsha, Hunan 410013, P.R. China
}

Received March 24, 2016; Accepted August 11, 2016

DOI: $10.3892 /$ or.2016.5182

\begin{abstract}
Osteosarcoma (OS) is an aggressive malignant tumor that is mesenchymal in origin with a very low 5-year survival rate, particularly in the patients with locally advanced or metastatic tumors and recurrent disease. MicroRNAs (miRNAs) play a critical role in essential biological processes as cellular proliferation, differentiation and apoptosis in normal or cancer cells, including OS cells. In the present study, we aimed to investigate the role of miR-422a in OS. We demonstrated that miR-422a expression was significantly downregulated in OS tissues and cell lines compared with the normal controls. In addition, overexpression of miR-422a was able to inhibit cell proliferation and the ability of invasion, and enhance paclitaxel and cisplatin-mediated apoptosis in OS cells. Inversely, downregulation of miR-422a exhibited an opposite role. We further demonstrated that miR-422a directly targeted TGF $\beta 2$ and regulated its expression and the activation of downstream molecules, $\operatorname{smad} 2$ and smad 3 in OS cells. Thus, miR-422a/TGF $\beta 2 /$ smad axis may be a potential target for OS treatment.
\end{abstract}

\section{Introduction}

Osteosarcoma (OS) is an aggressive malignant tumor that is mesenchymal in origin, which is of particular importance to children and adolescents as $\sim 60 \%$ of cases are pediatric patients (1). OS is characterized by highly complex heterogeneous tumor, by vast genomic instability and by multiple genomic aberrations at multiple chromosomes (2). Although the treatments, including surgery, radiation, chemotherapy and combination of them are improved, the 5-year survival rate of patients with OS

Correspondence to: Dr Mingjiang Liu, Department of Hand Surgery, Affiliated Nanhua Hospital, University of South China, 69 Chuanshan Road, Hengyang, Hunan 421002, P.R. China E-mail: minjliu2004@163.com

Key words: osteosarcoma, microRNA, chemosensitivity, transforming growth factor $\beta 2$, smad remains low, particularly in the patients with locally advanced or metastatic tumors, and recurrent disease (3). Thus, it is an urgent need to identify novel and targetable biomarkers for better diagnosis and prognosis of OS.

MicroRNAs (miRNAs) are a class of small non-coding RNA molecules, ranging between 18-25 nucleotides in length. They post-transcriptionally regulate target genes expression by binding at the $3^{\prime}$ untranslated region (3'UTR) (4). Each miRNA can control hundreds of gene transcripts. miRNAs play a critical role in essential biological processes such as cellular proliferation, differentiation and apoptosis in normal or cancer cells, including OS cells (5). miRNAs function as tumor suppressors or oncogenes depending on their target genes. Over the past few years, dysregulation of miRNAs has been implicated in pathogenesis and clinical relevance of OS (6). For example, Zhang et al found that miR-874 was significantly downregulated in OS cell lines and clinical specimens, and acted as an independent predictor of poor survival, which was associated with tumor size, distant metastasis and advanced clinical stage (7). miR-421 expression levels were upregulated in OS tissue and serum, and it may be a marker for diagnosis of OS (8). Recently, Niu et al found that transforming growth factor- $\beta 2$ (TGF $\beta 2$ ) was negatively regulated by miR-153, and overexpression of miR-153 decreased the expression of phosphorylated SMAD2 and SMAD3, the downstream molecules of TGF- $\beta$ in OS cell line MG-63 (9). miR-422a has been found to be tumor-suppressor in hepatocellular carcinoma (10). Furthermore, miR-422a was identified as discriminating miRNA in patient with OS, which could be easily transferable to diagnosis and may be a potent diagnostic tool to predict tumor sensitivity to chemotherapy drugs (11). The expression level of miR-422a was strongly decreased in OS bone tissue compared with non-cancerous bone tissues, which was associated with tumor size, advanced TNM stage, distant metastasis, grade of tumor and shorter overall survival time (12). However, the role of miR-422a in OS and the mechanisms by which miR-422a controls cell proliferation and apoptosis remain unknown.

In the present study, we aimed to investigate the role of miR-422a in OS. We analyzed the expression of miR-422a in OS tissues and cell lines. By gain and loss function 
experiments, we demonstrated that miR-422a was able to enhance paclitaxel and cisplatin induced apoptosis and functioned as a tumor suppressor in OS by directly targeting TGF 32 . Thus, we provide evidence to show that miR-422a may be potential therapy target for OS treatment.

\section{Materials and methods}

OS tissue samples. Ten adjacent tumor and $20 \mathrm{OS}$ tissues, and another 4 paired cancer and adjacent tissues, were collected from Hunan Cancer Hospital. Informed consents have been signed by all subjects. All samples were collected and identified by histopathological evaluation, and stored at $-80^{\circ} \mathrm{C}$ until used.

Cell culture. Human OS cell lines MG-63, Saos-2 and U-2 0S, and normal human fetal osteoblast hFOB cells were obtained from the American Type Culture Collection (ATCC; Manassas, VA, USA). All the cells were cultured in Dulbecco's modified Eagle's medium (DMEM), supplemented with $10 \%(\mathrm{v} / \mathrm{v})$ fetal bovine serum (FBS), $100 \mathrm{U} / \mathrm{ml}$ penicillin/streptomycin (all from Invitrogen Life Technologies, Carlsbad, CA, USA) at $37^{\circ} \mathrm{C}$ in a humidified $5 \% \mathrm{CO}_{2}$ incubator.

Cell treatment. To knock down or overexpress the expression of miR-422a, the cells were transfected with pre-miR-422a or anti-miR-422a using Lipofectamine 2000 (Life Technologies) according to the manufacturer's instructions. The cells transfected with scramble inhibitor or mimics were used as negative control.

To suppress TGF $\beta /$ smad signaling, TGF $\beta$ inhibitor SB431542 (0.3 $\mu \mathrm{M}$; Sigma-Aldrich, St. Louis, MO, USA) was added to the cultured medium.

Quantitative PCR ( $q P C R$ ) analysis. TRIzol reagent (Invitrogen, Carlsbad, CA, USA) was used to extract total RNA from the indicated cells according to the manufacturer's instructions. SYBR-Green qRCR mix (Toyobo, Shanghai, China) was used for real-time PCR to detect mRNA levels. The primers were as follows: TGF $\beta 2$ sense, AGCAAGCT GAAGCTCACCAGT and antisense, TTGGCGTAGTACTCT TCGTCG; $\beta$-actin sense, AGGGGCCGGACTCGTCATACT and antisense, GGCGGCACCACCATGTACCCT. miRNA qRT-PCR Starter kit (RiboBio, Guangzhou, China) was used for real-time PCR to detect miR-422a levels. The primers of miR-422a (HmiRQP0490) and U6 were purchased from FulenGen (Guangzhou, China). The expression of target genes was normalized by $\beta$-actin or U6. All the qPCR data were processed using $2^{-\Delta \Delta C t}$ method.

Western blot analysis. The protein was extracted by RIPA lysis buffer (Auragene, Changsha, China) from indicated cells, and Bradford protein assay kit (Beyotime Biotechnology, Shanghai, China) was used to measure the protein concentration. After separated on 10\% SDS-PAGE gels, the protein was transferred to nitrocellulose membranes. The membranes were blocked with 5\% non-fat dry milk and incubated with primary antibodies mouse monoclonal anti-TGF 32 (cat no. ab36495; dilution: 1:1,000), rabbit polyclonal anti-smad2 (cat no. ab17812; dilution: 1:1,000) and rabbit polyclonal anti-smad3 (cat no. ab122028; dilution: 1:1,000) were purchased from Abcam (Cambridge, UK), goat polyclonal anti-phosphorylated smad3 and phosphorylated smad2 (1:500) from Santa Cruz Biotechnology (Dallas, TX, USA), mouse monoclonal anti- $\beta$-actin $(1: 5,000)$ from Sigma-Aldrich overnight at $4^{\circ} \mathrm{C}$. The membranes were washed with Tris-buffered saline with Tween-20 (TBST), and were then incubated with appropriate horseradish peroxidase (HRP)-conjugated secondary antibody. Enhanced chemiluminescence (ECL) reagent was used to detect the signal on the membrane.

Dual-luciferase report system. Wild-type (wt) and mutant (mut) 3'UTR of TGF 32 were designed by FulenGen, which was inserted into the downstream of dual-luciferase reporter vector, and the mutant sites are shown in Fig. 6A. For luciferase assay, $5 \times 10^{4}$ cells were plated and cultured into 24 -well plates to reach $\sim 70 \%$ confluency. U-2 OS cells were co-transfected with miR-422a mimics or miR-422a inhibitors and wt/mut 3'UTR of TGF $\beta 2$ dual-luciferase reporter vector, respectively. After $48 \mathrm{~h}$ transfection, the luciferase activity was detected using Dual-Luciferase reporter gene assay kit on luminometer (E9031) (both from Promega, Madison, WI, USA).

MTT assay. Cell growth was measured by MTT assay. Indicated cells $(2,000)$ were seeded in each 96 -well plate for $12 \mathrm{~h}$, and further incubated for $0,24,48$ and $72 \mathrm{~h}$, respectively. One hour before the end of incubation, $100 \mu 1$ MTT at $5 \mathrm{mg} /$ $\mathrm{ml}$ final concentration were added to each well for $4 \mathrm{~h}$ at $37^{\circ} \mathrm{C}$. Then, the supernatant were removed and $150 \mu l$ dimethyl sulfoxide (DMSO) was added to each well for $15 \mathrm{~min}$, and optical density (OD) $570 \mathrm{~nm}$ value in each well was determined by an enzyme immunoassay analyzer.

Flow cytometric analysis of apoptosis. Cells from each group were trypsinized and washed with cold phosphate-buffered saline (PBS), and Annexin V-FITC/PI (KeyGen, Nanjing, China) were then used to stain the apoptotic cells according to the manufacturer's instructions. The apoptosis rate was analyzed by flow cytometry (MoFlo XDP; Beckman Coulter, Brea, CA, USA). The experiments were independently performed in triplicate.

Transwell assay. The indicated cells were starved for $24 \mathrm{~h}$, and then resuspended in serum-free medium and added to the upper chamber of Transwell (Becton-Dickinson Company, Franklin Lakes, New Jersey, USA). The lower chamber was filled with completed medium containing 10\% FBS. Following $48 \mathrm{~h}$ culture, cells attached to the bottom were fixed and stained with crystal violet for $30 \mathrm{~min}$. The OD at $570 \mathrm{~nm}$ of crystal violet dissolved by $10 \%$ acetic acid was detected by an enzyme immunoassay analyzer (MK3; Thermo Fisher Scientific, Waltham, MA, USA).

Statistical analysis. Statistical analyses were performed using GraphPad Prism 5 software (Graphpad Software, Inc., La Jolla, CA, USA) and the data are presented as the mean \pm standard deviation. An unpaired two-tailed Student's t-test or one way analysis of variance (ANOVA) with Bonferroni post hoc test was used to analyze the data depending on conditions. $\mathrm{P}<0.05$ was considered to indicate a statistically significant difference. 


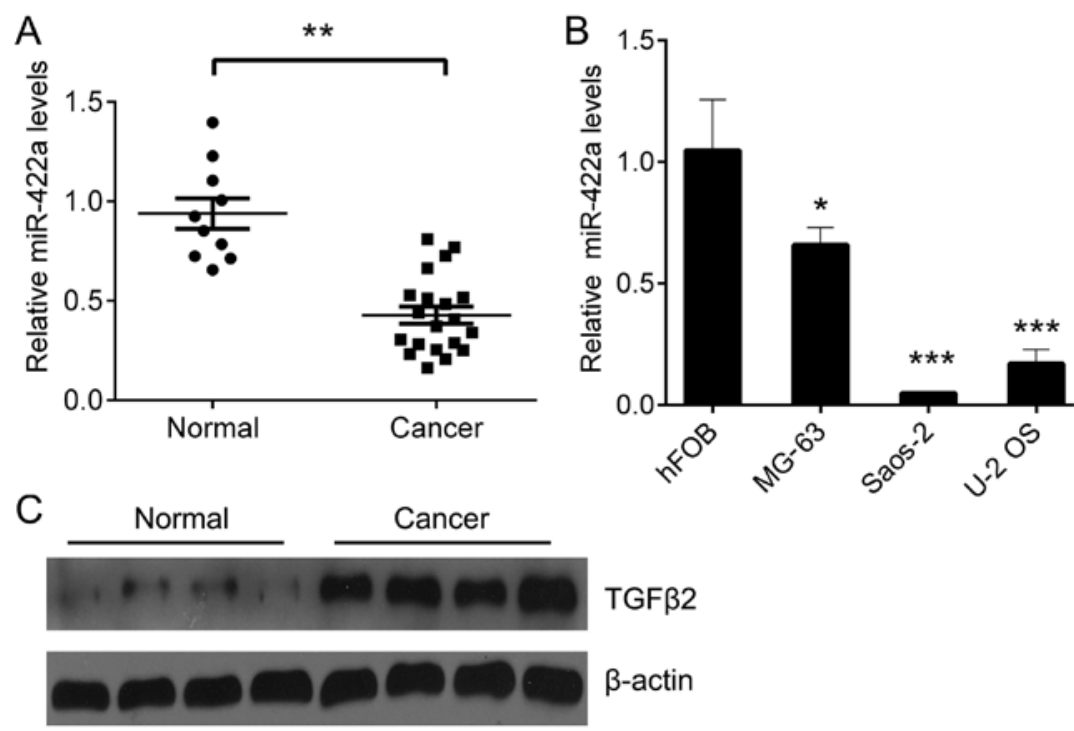

Figure 1. The expression of miR-422a and TGF 32 in OS tissues and cell lines. (A) qPCR analysis for miR-422a in OS and adjacent tissues. (B) qPCR analysis for miR-422a in OS cell lines. (C) Western blot analysis for TGF $\beta 2$ in 4 paired adjacent and OS tissues. Data are presented as means $\pm \mathrm{SD}$. "P<0.05, ${ }^{* *} \mathrm{P}<0.01$, ${ }^{* * *} \mathrm{P}<0.001$.

\section{Results}

The expression of $\mathrm{miR}-422 a, T G F \beta 2$ in $O S$ tissues and cell lines. To investigate the role of miR-422a in OS, we collected $20 \mathrm{OS}$ and 10 adjacent tissues to detect the expression of miR-422a using qPCR. Our data showed that the average expression level of miR-422a was significantly downregulated in OS tissue samples compared with adjacent controls (Fig. 1A). In addition, similar results were observed in the human OS cell lines, the expression of miR-422a in OS cell lines was lower than in hFOB cells (Fig. 1B). In addition, we also analyzed the expression of the putative targets of miR-422a, TGF $\beta 2$ in OS tissue samples. We found that the protein levels of TGF $\beta 2$ were significantly upregulated in 4 paired OS and normal adjacent tissues (Fig. 1C). These results indicate that miR-422a and TGF $\beta 2$ play an important role in OS.

The effects of miR-422a on cell proliferation and invasion in $U-2$ OS and MG-63 cells. To investigate the role of miR-422a in OS, we overexpressed or knocked down the expression of miR-422a in U-2 OS and MG-63 cells (Fig. 2A). We found that overexpression of miR-422a inhibited cell proliferation, while downregulation of miR-422a promoted cell proliferation in U-2 0S and MG-63 cell lines (Fig. 2B). In addition, upregulation of miR-422a significantly increased apoptosis, whereas downregulation of miR-422a exhibited an opposite role (Fig. 3A). Furthermore, we also accessed the role of miR-422a in cell invasion. Transwell assay was used to analyze cell invasion after overexpression or knockdown of miR-422a. Upregulation of miR-422a significantly reduced invasion ability compared with the negative control, while knockdown of miR-422a was able to enhance the ability of cell invasion in U-2 OS and MG-63 cell lines (Fig. 3B). Thus, miR-422a functions as a tumor suppressor in OS.

miR-422a sensitize OS cells to paclitaxel and cisplatin. Furthermore, we investigated whether miR-422a had synergic effects with current chemotherapy drug used in OS. We found that overexpression of miR-422a significantly decreased the $\mathrm{IC}_{50}$ values of paclitaxel (3.4 vs. $\left.6.2 \mathrm{mg} / \mathrm{l}\right)$ and cisplatin (8.6 vs. $11.9 \mu \mathrm{M}$ ) compared with negative control, while downregulation of miR-422a significantly increased the $\mathrm{IC}_{50}$ values of paclitaxel (6.2 vs. $8.0 \mathrm{mg} / \mathrm{l})$ and cisplatin (14.7 vs. $11.8 \mu \mathrm{M})$ compared with negative control in U-2 0S cells (Fig. 4A). Similar results were also observed in MG-63 cells (Fig. 4B). The $\mathrm{IC}_{50}$ values of paclitaxel and cisplatin were decreased from 6.3 to $5 \mathrm{mg} / \mathrm{l}$, and from 11.5 to $7.9 \mu \mathrm{M}$, respectively, in MG-63 cells overexpressing miR-422a. In contrast, the $\mathrm{IC}_{50}$ values of paclitaxel and cisplatin were increased from 6.2 to $8.1 \mathrm{mg} / 1$, and from 11.2 to $17.4 \mu \mathrm{M}$, respectively, by downregulation of miR-422a in MG-63 cells. In addition, upregulation of miR-422a significantly enhanced paclitaxel and cisplatin-mediated apoptosis in U-2 0S and MG-63 cells, whereas downregulation of miR-422a exhibited an opposite role (Fig. 5). Thus, miR-422a is able to sensitize OS cells to chemotherapy drugs paclitaxel and cisplatin.

miR-422a regulates TGF $\beta 2$ expression at transcriptional and translational levels by directly targeting its 3'UTR. To further investigate the downstream molecules targeted by miR-422a, we screened the putative targets of miR-422a using bioinformatic tool (http://www.microrna.org) and found that miR-422a conversely targeted to TGF $\beta 2$. We performed dualluciferase report gene assay in U-2 0 S cells to confirm whether the 3'UTR of TGF $\beta 2$ had a direct target site for miR-422a. The sequences that containing the wt or mut 3'UTR of TGF 32 were constructed into dual-luciferase reporter gene (Fig. 6A). We found that the luciferase activity was significantly repressed in the pre-miR-422a transfectant compared with the negative control transfectant. Moreover, miR-422a-mediated repression of luciferase activity was abolished by the mutant type 3'UTR of TGF $\beta 2$ (Fig. 6B). Moreover, the mRNA and protein levels of TGF $\beta 2$ were markedly downregulated by pre-miR-422a transfection, and upregulated by anti-miR-422a 

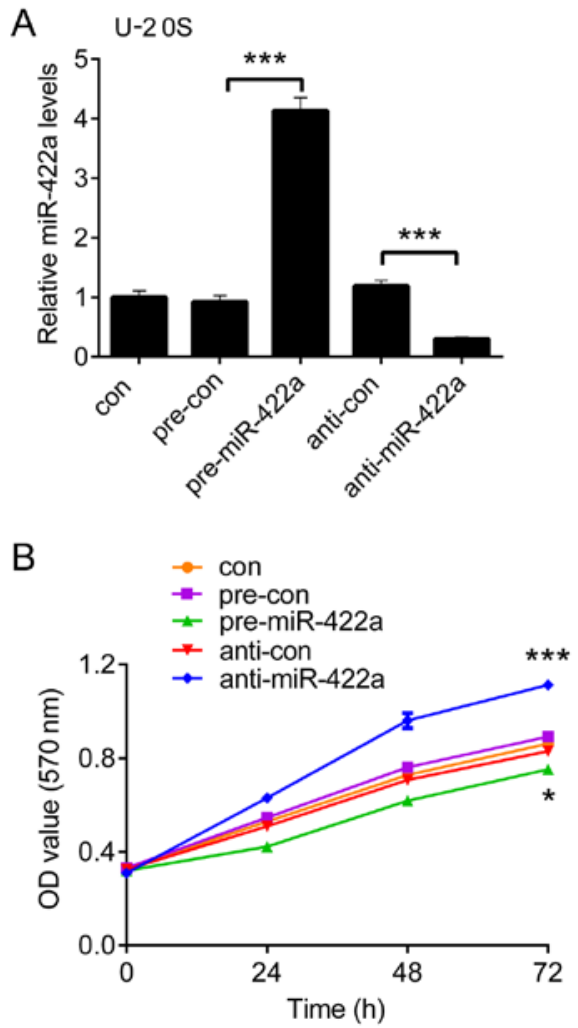
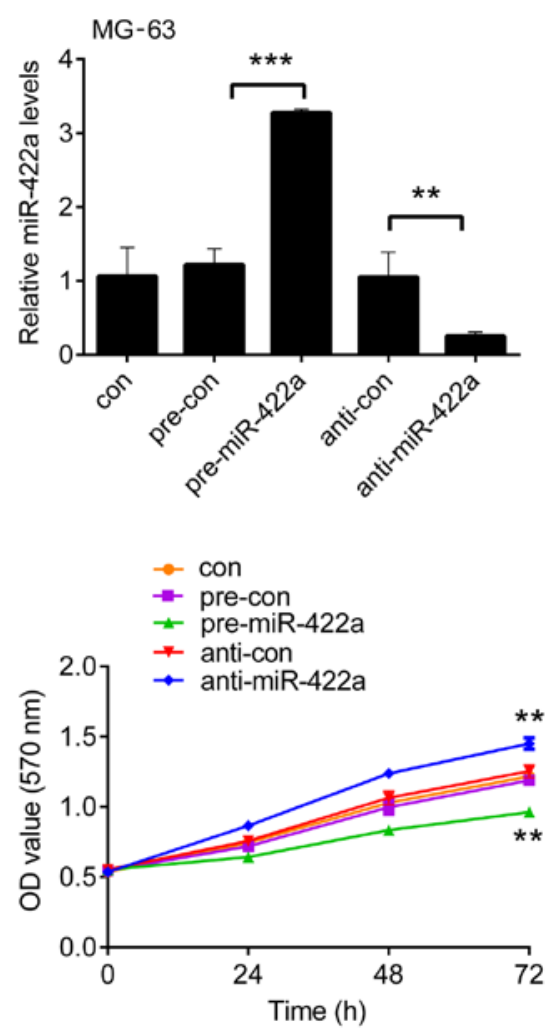

Figure 2. The effects of miR-422a on cell proliferation (A) qPCR analysis for miR-422a in U-2 0S cells (left) and MG-63 cells (right) after transfection with pre-miR-422a or anti-miR-422a. (B) MTT assay was used to measure cell proliferation in U-2 0S cells (left) and MG-63 cells (right) after transfection with pre-miR-422a or anti-miR-422a. Data are presented as means $\pm \mathrm{SD} ;{ }^{*} \mathrm{P}<0.05,{ }^{* *} \mathrm{P}<0.01,{ }^{* * *} \mathrm{P}<0.001$.
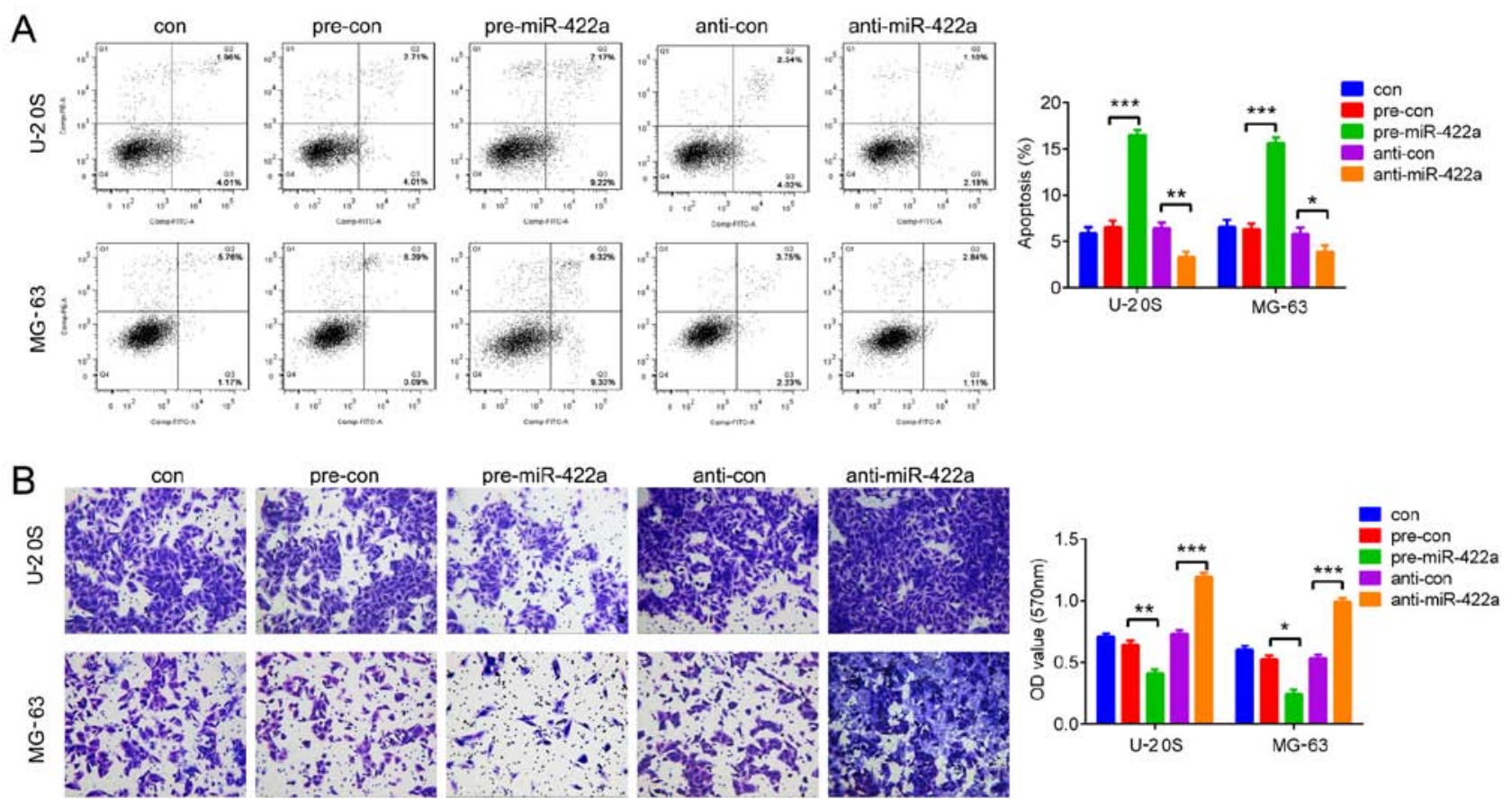

Figure 3. The effects of miR-422a on cell apoptosis and invasion. (A) Flow cytometric analysis was used to measure cell apoptosis in U-2 0S cells (left) and MG-63 cells (right) after transfection with pre-miR-422a or anti-miR-422a. (B) Transwell assay was used to analyze cell invasion in U-2 0S cells (left) and MG-63 cells (right) after transfection with pre-miR-422a or anti-miR-422a. Data are presented as means $\pm \mathrm{SD} ;{ }^{*} \mathrm{P}<0.05,{ }^{* *} \mathrm{P}<0.01,{ }^{* * *} \mathrm{P}<0.001$.

transfection compared with the negative control, respectively. These results determine that miR-144 directly targets TGF $\beta 2$ and regulates its expression at transcriptional and translational levels. Furthermore, we tested the downstream molecules of 

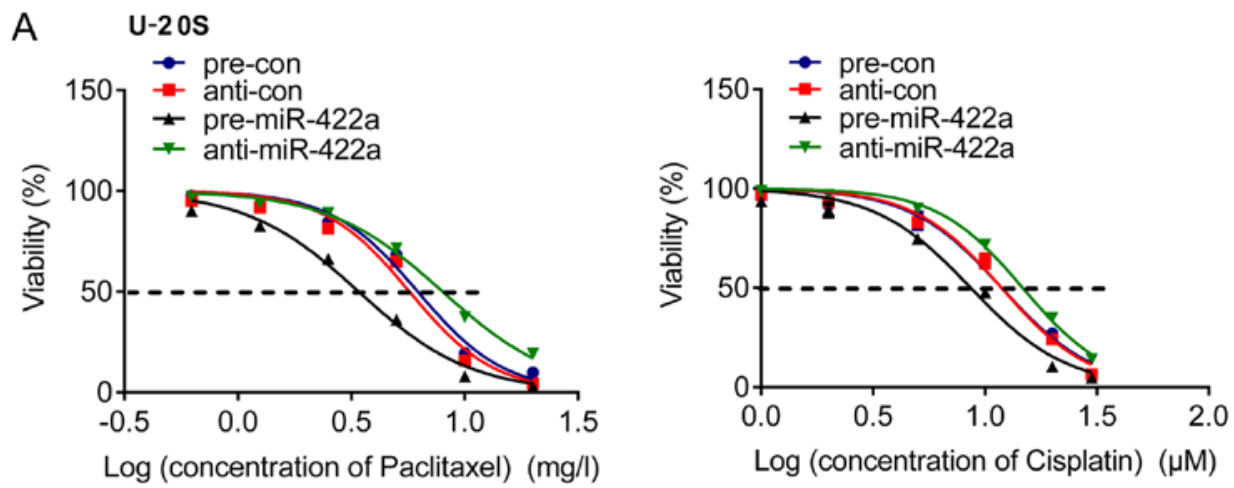

B
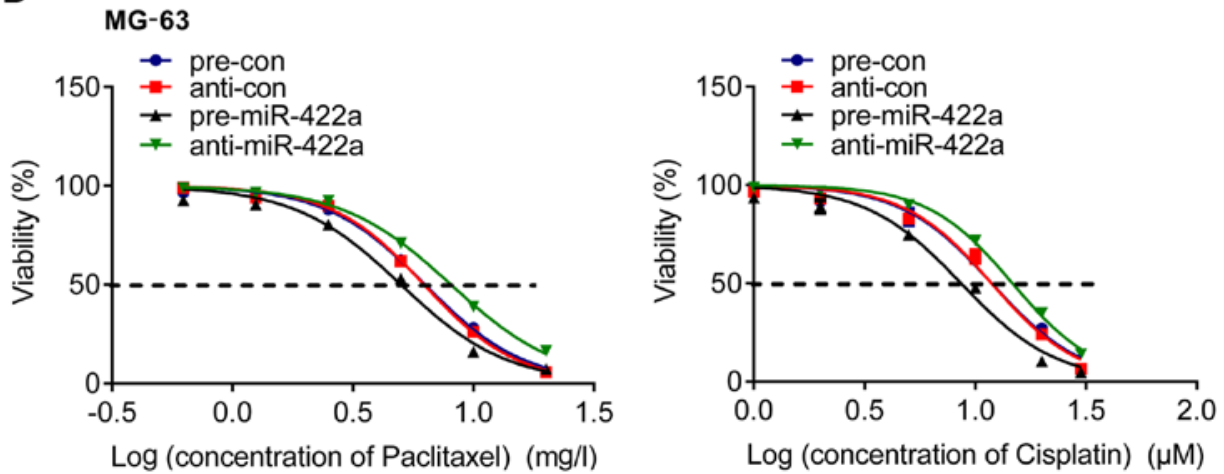

Figure 4. Overexpression of miR-422a sensitizes U-2 OS and MG-63 cells to paclitaxel and cisplatin (A) U-2 0S and (B) MG-63 cells were transfected with pre-miR-422a or anti-miR-422a for $48 \mathrm{~h}$ and then were treated with a range of paclitaxel (left) or cisplatin (right). MTT assay was used to evaluate cell viability.

A

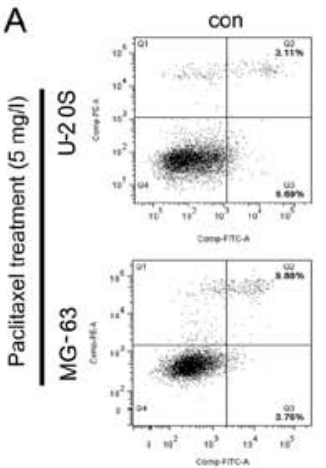

B

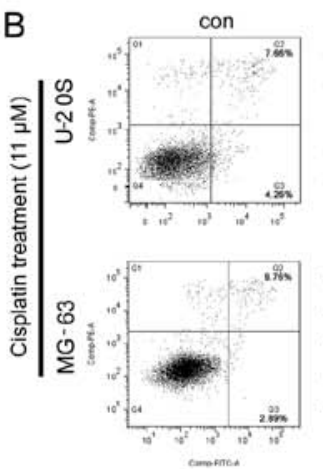

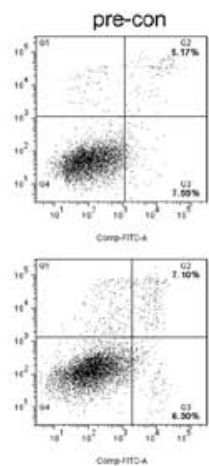
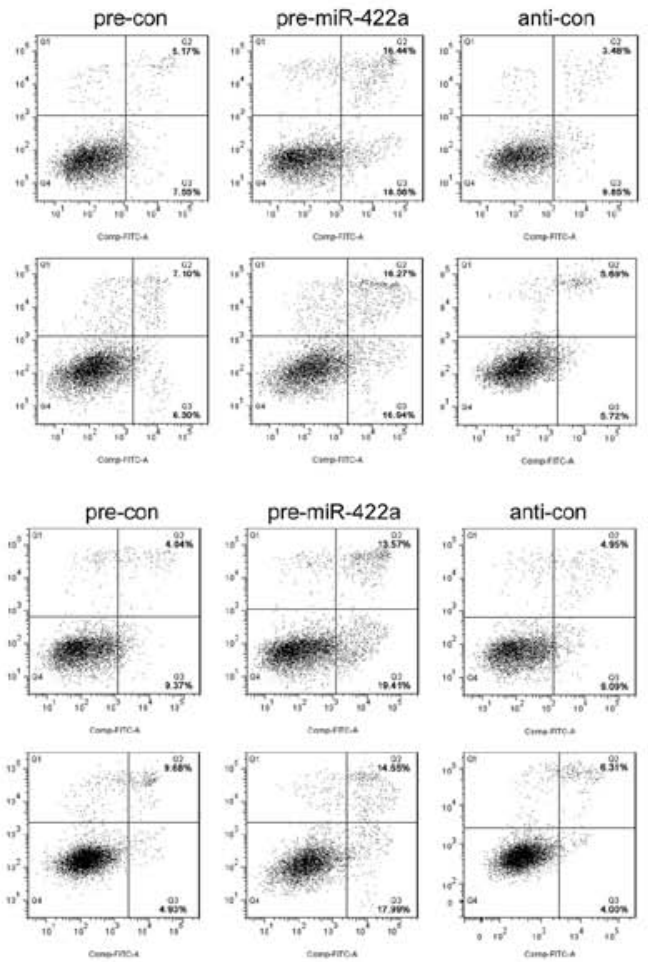
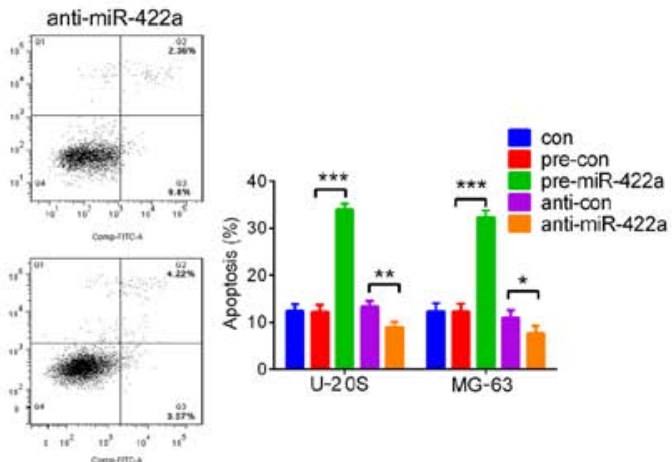

Figure 5. Overexpression of miR-422a enhances apoptosis induced by paclitaxel and cisplatin in U-2 0S and MG-63 cells. U-2 0S and MG-63 cells were transfected with pre-miR-422a or anti-miR-422a for $48 \mathrm{~h}$, and were then treated with (A) paclitaxel or (B) cisplatin. Flow cytometric analysis was used to evaluate cell apoptosis. Data are presented as means $\pm \mathrm{SD} ;{ }^{*} \mathrm{P}<0.05,{ }^{* *} \mathrm{P}<0.01,{ }^{* * * *} \mathrm{P}<0.001$.

TGF 32 smad2 and smad 3 by western blotting in U-2 OS and MG-63 cells after miR-422a knockdown or overexpression.
Although overexpression and knockdown of miR-422a did not alter the expression of total $\operatorname{smad} 2 / 3$, a significant decrease of 
A

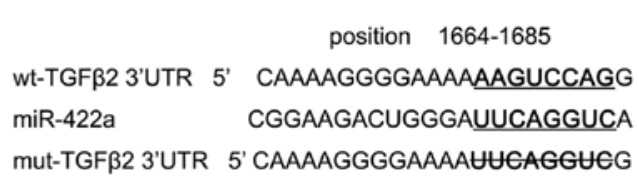

C
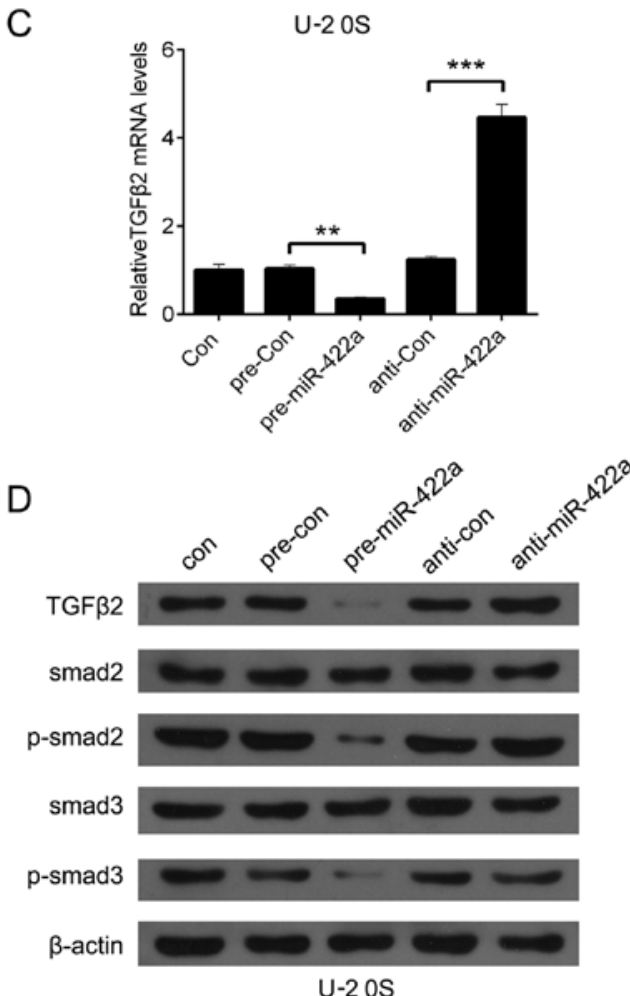
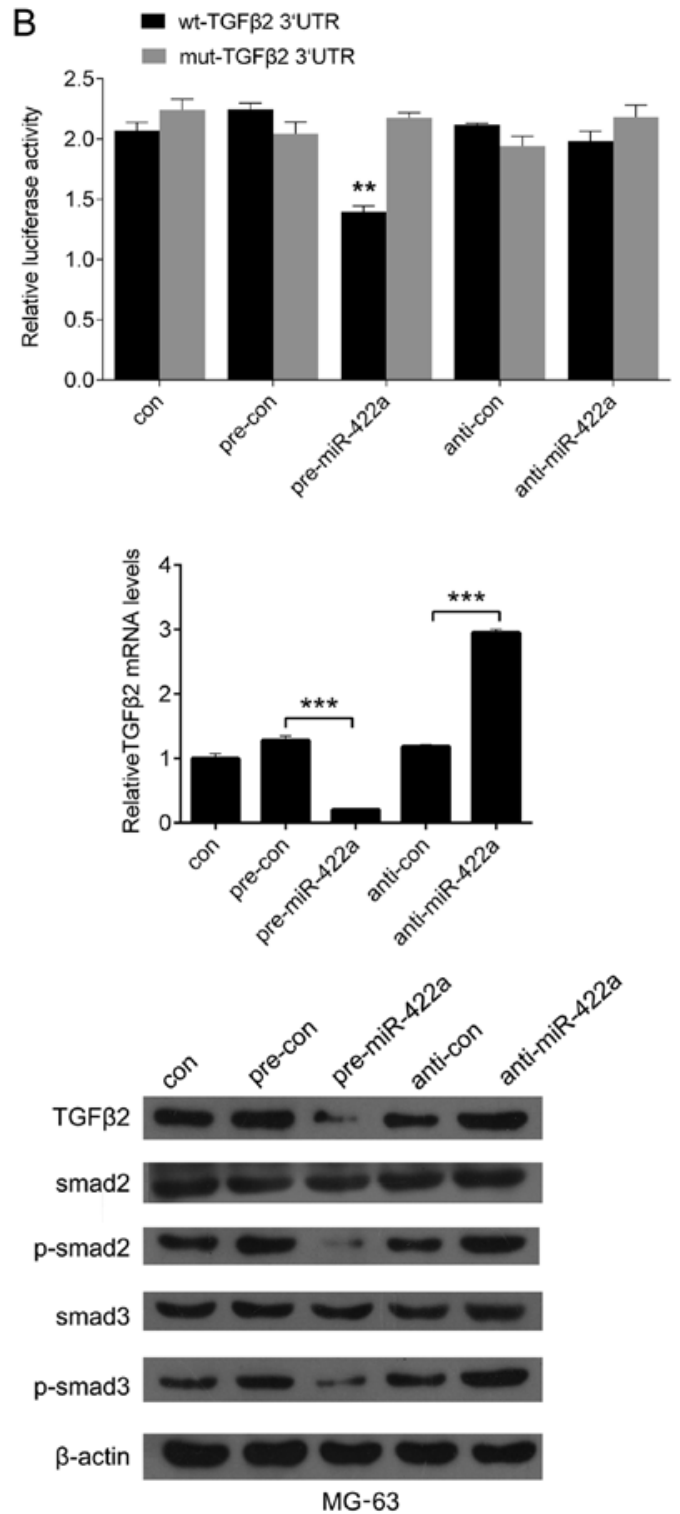

Figure 6. miR-422a regulates TGF 32 expression at transcriptional and translational levels. (A) The target sites (with underline) and the mutant site (with line-through) at 3'UTR of TGF 32 . (B) U-2 OS cells were co-transfected with miR-422a mimics/inhibitor and wt-3'UTR of TGF $\beta 2$ or their mutant type, respectively. Compared with negative control, the luciferase activity of wt-TGF $\beta 2$ was deceased significantly by miR-422a, which was abrogated by their mutant type. The luciferase activity had no significant changes in cells treated with miR-422a inhibitor. (C) qPCR analysis for TGF 32 in U-2 0S cells (left) and MG-63 cells (right) treated with pre-miR-422a mimics or anti-miR-422a. (D) Western blot analysis for TGF 32 and smad2/3 in U-2 0S cells (left) and MG-63 cells (right) treated with pre-miR-422a mimics or anti-miR-422a. Data are presented as means $\pm \mathrm{SD} ;{ }^{* * *} \mathrm{P}<0.01,{ }^{* * * *} \mathrm{P}<0.001$.

phosphorylated smad2 and smad3 were observed in U-2 OS and MG-63 cells with upregulated miR-422a; and knockdown of miR-422a significantly increased the expression of phosphorylated smad 2 and smad 3 compared with the negative control (Fig. 6D). Moreover, we inhibited the TGF $32 / \mathrm{smad}$ signaling using TGF $\beta 2$ inhibitor SB431542. We found that TGF 32 inhibitor SB431542 was able to inhibit the ability of migration and invasion, and enhance the effects of miR-422a on cell migration and invasion (Fig. 7). These results suggest that miR-422a inhibits the ability of migration and invasion of OS cells through the TGF $\beta 2 /$ smad signaling.

\section{Discussion}

In the present study, we demonstrated that miR-422a expression was significantly downregulated in osteosarcoma (OS) tissues and cell lines compared with the normal controls. In addition, overexpression of miR-422a was able to inhibit cell proliferation and the ability of invasion and enhance paclitaxel and cisplatin-mediated apoptosis in OS cells. These results suggest that miR-422a functions as a tumor suppressor in OS.

Downregulated miR-422a has been found in several types of cancers, including OS, hepatocellular carcinoma and colorectal cancer $(10,12,13)$. The low expression levels of miR-422a is correlated with large tumor size, advanced TNM stage, distant metastasis, high grade of tumor and shorter time overall survival, suggesting that miR-422a is an independent prognostic marker of overall survival of patients with OS (12). miR-422a is differentially expressed in the HCC patients serum, and is significantly associated with the patients relapse-free survival (RFS). However, the expression levels of miR-422a are positively correlated in HCC tumor tissues and 


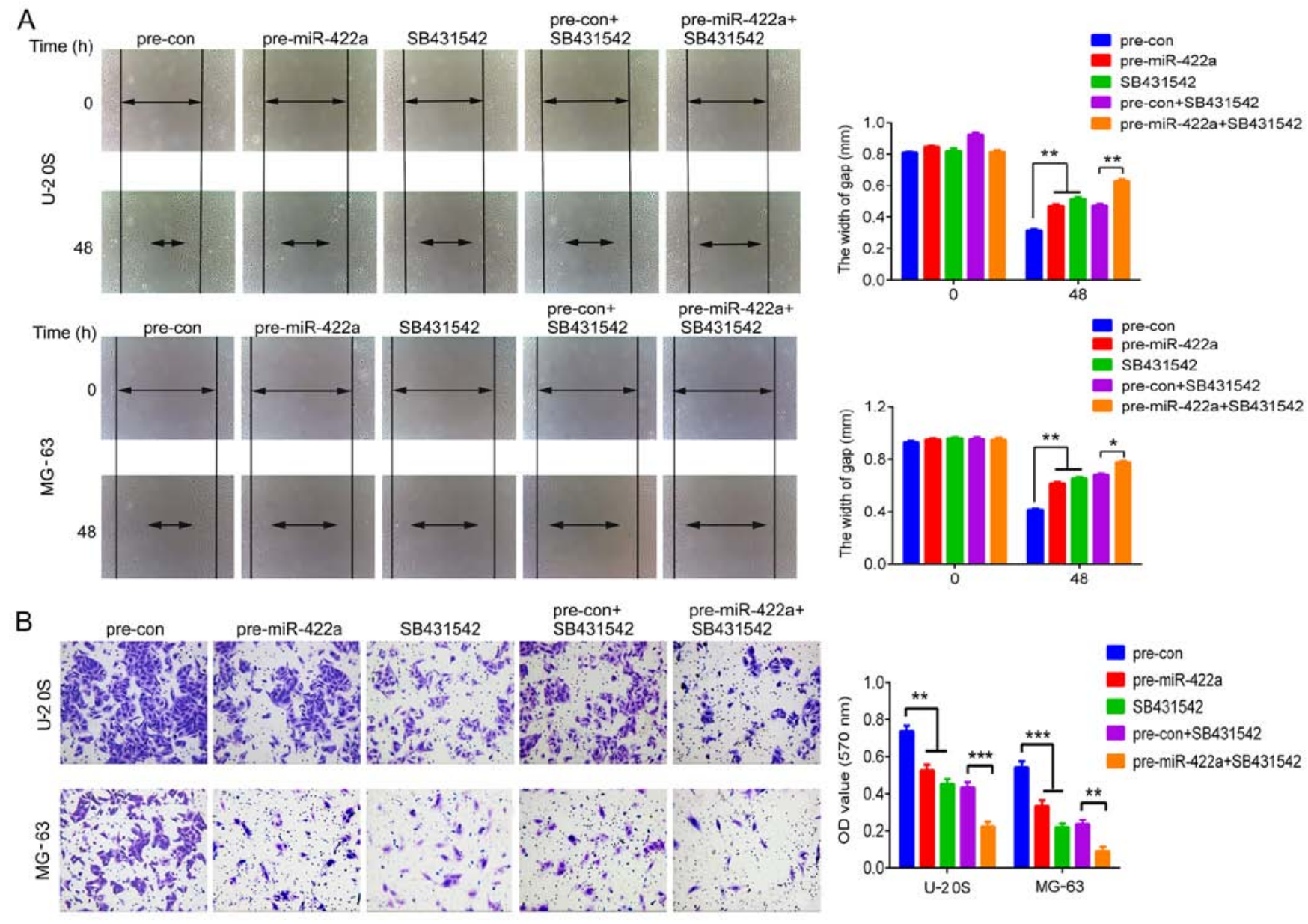

Figure 7. TGF 32 inhibitor enhances the effects of miR-422a on cell migration and invasion. (A) Scratch assay was used to measured cell migration in U-2 0S cells (upper) and MG-63 cells (low) after indicated treatment. (B) Transwell assay was used to analyze cell invasion in U-2 0S cells (upper) and MG-63 cells (low) after indicated treatment. Data are presented as means $\pm \mathrm{SD} ;{ }^{*} \mathrm{P}<0.05,{ }^{* *} \mathrm{P}<0.01,{ }^{* * *} \mathrm{P}<0.001$.

the paired serum samples (14). In addition, Zhang et al reports that downregulated miR-422a is correlated with pathological grading, recurrence and metastasis in HCC. The restoration of miR-422a expression in HCC tumor cells significantly inhibits cell proliferation and migration in vitro and in vivo through the double-negative feedback loop between miR-422a and its targets forkhead box G1 (FOXG1), FOXQ1 and FOXE1 (10). The downregulation of miR-422a was associated with a poorer prognosis in colorectal cancer. It seems that miR-422a interacts with human telomerase reverse transcriptase and inhibits its expression to function as a tumor suppressor in colorectal cancer (15), and serum microRNA panel containing miR-422a may have a high diagnostic accuracy of colorectal adenocarcinoma (16). However, miR-422a is found to be upregulated in pituitary adenomas including non-functioning and gonadotropin-secreting (17), indicating that miR-422a is a cancer specific gene.

Gougelet et al showed that miR-422a can discriminate good from bad respond to ifosfamide in OS of rat and human origins, which may target the TGF $\beta$, the Wnt and the MAP kinase pathways (11). We now demonstrate that miR-422a directly targets TGF $\beta 2$ and regulates its expression and the activation of downstream molecules, smad 2 and $\operatorname{smad} 3$ in OS cells. TGF- $\beta /$ smad signaling frequently promotes cancers in cooperation with other transcription factors or through crosstalk with other signaling pathways including extracellular signal-regulated kinase (ERK) signaling, p38 mitogen-activated protein kinases (MAPKs) and phosphoinositide 3-kinase (PI3K)/AKT pathways (18). The canonical TGF $/$ Smad signaling transmits signal via binding to two related transmembrane receptors, which subsequently phosphorylate receptor-regulated Smad proteins, Smad2 and Smad3, the canonical TGF- $\beta$ effector molecules (19). Smad2 and Smad3 phosphorylation triggers their activation to regulate gene expression in a cell type-specific manner (20). TGF $\beta 2$ is able to trigger the malignant phenotype in human OS cells, indicating that TGF $\beta 2$ may account for the metastatic potential of these cells (21). In addition, Saos- 2 cell adhesion is dependent on active TGF- $\beta 2$ signaling (22). The TGF- $\beta$ kinase inhibitor LY2109761 is sufficient to induce apoptosis, inhibit the ability of invasion in MG-63 cells, and combination with LY2109761 significantly augments the cytotoxicity of cisplatin in MG-63 cells (23). In addition, the serum level of TGF- $\beta$ is significantly increased in patients with OS compared to healthy controls. Furthermore, patients with metastasis have augmented levels of serum TGF- $\beta$ than those without metastasis. These data indicate that TGF- $\beta$ may be closely involved in the pathogenesis of OS (24). In addition to miR-422a, it was 
reported that transforming growth factor- $\beta 2$ (TGF $\beta 2$ ) was also negatively regulated by miR-153, a tumor suppressor in OS. In addition, overexpression of miR-153 decreased the expression of downstream signaling molecules of TGF- $\beta$, p-SMAD2 and p-SMAD3 (10). In the present study, we found that TGF $\beta 2$ inhibitor SB431542 was able to inhibit the ability of migration and invasion, and enhance the effects of miR-422a on cell migration and invasion, suggesting that miR-422a inhibits the ability of migration and invasion of OS cells through the TGF $32 /$ smad signaling.

In conclusion, the present study demonstrates that overexpression of miR-422a decreases TGF $\beta 2$ expression by directly binding to its $3^{\prime} \mathrm{UTR}$, resulting in downstream effector modulation including phosphorylated smad 2 and smad 3 to regulate OS proliferation and invasion, and to enhance paclitaxel and cisplatin-mediated apoptosis. Thus, miR-422a/TGF $\beta 2 /$ smad axis may be a potential target for OS treatment.

\section{References}

1. Sampson VB, Yoo S, Kumar A, Vetter NS and Kolb EA: MicroRNAs and potential targets in osteosarcoma: Review. Front Pediatr 3: 69, 2015.

2. Lim HJ and Yang JL: Regulatory roles and therapeutic potential of microRNA in sarcoma. Crit Rev Oncol Hematol 97: 118-130, 2016.

3. Varshney $\mathbf{J}$ and Subramanian S: MicroRNAs as potential target in human bone and soft tissue sarcoma therapeutics. Front Mol Biosci 2: 31, 2015.

4. Zhou G, Shi X, Zhang J, Wu S and Zhao J: MicroRNAs in osteosarcoma: From biological players to clinical contributors, a review. J Int Med Res 41: 1-12, 2013.

5. Zhou W, Hao M, Du X, Chen K, Wang G and Yang J: Advances in targeted therapy for osteosarcoma. Discov Med 17: 301-307, 2014.

6. Zhang J, Yan YG, Wang C, Zhang SJ, Yu XH and Wang WJ: MicroRNAs in osteosarcoma. Clin Chim Acta 444: 9-17, 2015.

7. Zhang LQ, Sun SL, Li WY, Feng Z, Xu XY, Zhuang QS and Fang J: Decreased expression of tumor suppressive miR-874 and its clinical significance in human osteosarcoma. Genet Mol Res 14: 18315-18324, 2015.

8. Zhou S, Wang B, Hu J, Zhou Y, Jiang M, Wu M, Qin L and Yang X: miR-421 is a diagnostic and prognostic marker in patients with osteosarcoma. Tumour Biol: Jan 13, 2016 (Epub ahead of print).

9. Niu G, Li B, Sun L and An C: MicroRNA-153 inhibits osteosarcoma cells proliferation and invasion by targeting TGF- $\beta 2$. PLoS One 10: e0119225, 2015.

10. Zhang J, Yang Y, Yang T, Yuan S, Wang R, Pan Z, Yang Y, Huang G, Gu F, Jiang B, et al: Double-negative feedback loop between microRNA-422a and forkhead box (FOX)G1/Q1/E1 regulates hepatocellular carcinoma tumor growth and metastasis. Hepatology 61: 561-573, 2015.
11. Gougelet A, Pissaloux D, Besse A, Perez J, Duc A, Dutour A Blay JY and Alberti L: Micro-RNA profiles in osteosarcoma as a predictive tool for ifosfamide response. Int J Cancer 129: 680-690, 2011.

12. Bahador R, Taheriazam A, Mirghasemi A, Torkaman A, Shakeri M, Yahaghi E and Goudarzi PK: Tissue expression levels of miR-29b and miR-422a in children, adolescents, and young adults' age groups and their association with prediction of poor prognosis in human osteosarcoma. Tumour Biol 37: 3091-3095, 2016.

13. Faltejskova P, Svoboda M, Srutova K, Mlcochova J, Besse A, Nekvindova J, Radova L, Fabian P, Slaba K, Kiss I, et al: Identification and functional screening of microRNAs highly deregulated in colorectal cancer. J Cell Mol Med 16: 2655-2666, 2012.

14. Wang L, Liu M, Zhu H, Rong W, Wu F, An S, Liu F, Feng L, Wu J and Xu N: Identification of recurrence-related serum microRNAs in hepatocellular carcinoma following hepatectomy. Cancer Biol Ther 16: 1445-1452, 2015

15. Qin YZ, Xie XC, Liu HZ, Lai H, Qiu H and Ge LY: Screening and preliminary validation of miRNAs with the regulation of hTERT in colorectal cancer. Oncol Rep 33: 2728-2736, 2015.

16. Zheng G, Du L, Yang X, Zhang X, Wang L, Yang Y, Li J and Wang C: Serum microRNA panel as biomarkers for early diagnosis of colorectal adenocarcinoma. Br J Cancer 111: 1985-1992, 2014

17. Liang S, Chen L, Huang $\mathrm{H}$ and Zhi D: The experimental study of miRNA in pituitary adenomas. Turk Neurosurg 23: 721-727, 2013.

18. Won KY, Kim YW and Park YK: Expression of Smad and its signalling cascade in osteosarcoma. Pathology 42: 242-247, 2010.

19. Johnsen SA, Subramaniam M, Janknecht R and Spelsberg TC: TGFbeta inducible early gene enhances TGFbeta/Smad-dependent transcriptional responses. Oncogene 21: 5783-5790, 2002.

20. Zaidi SK, Sullivan AJ, van Wijnen AJ, Stein JL, Stein GS and Lian JB: Integration of Runx and Smad regulatory signals at transcriptionally active subnuclear sites. Proc Natl Acad Sci USA 99: 8048-8053, 2002.

21. Nikitovic D, Zafiropoulos A, Katonis P, Tsatsakis A, Theocharis AD, Karamanos NK and Tzanakakis GN: Transforming growth factor-beta as a key molecule triggering the expression of versican isoforms $\mathrm{v} 0$ and $\mathrm{v} 1$, hyaluronan synthase-2 and synthesis of hyaluronan in malignant osteosarcoma cells. IUBMB Life 58: 47-53, 2006.

22. Nikitovic D, Chalkiadaki G, Berdiaki A, Aggelidakis J, Katonis P, Karamanos NK and Tzanakakis GN: Lumican regulates osteosarcoma cell adhesion by modulating TGF $\beta 2$ activity. Int J Biochem Cell Biol 43: 928-935, 2011.

23. Ren XF, Mu LP, Jiang YS, Wang L and Ma JF: LY2109761 inhibits metastasis and enhances chemosensitivity in osteosarcoma MG-63 cells. Eur Rev Med Pharmacol Sci 19: 1182-1190, 2015.

24. Xu S, Yang S, Sun G, Huang W and Zhang Y: Transforming growth factor-beta polymorphisms and serum level in the development of osteosarcoma. DNA Cell Biol 33: 802-806, 2014. 